European Journal of Neuroscience, Vol. 41, pp. 1484-1495, 2015 (author's post-print version)

\title{
The effects of cross-orientation masking on the visual gamma response in humans
}

Gavin Perry

1. Cardiff University Brain Imaging Centre (CUBRIC), School of Psychology, Cardiff University, 70 Park Place, Cardiff, CF10 3AT.

2. Institute of Psychological Medicine and Clinical Neurosciences, School of Medicine, Cardiff University, Hadyn Ellis Building, Maindy Road, Cardiff, CF24 4HQ.

Correspondence: Gavin Perry, CUBRIC, School of Psychology, 70 Park Place, Cardiff, CF10 3AT. Email:perryg@cardiff.ac.uk. Fax:+44 (0)29 20870339.

Keywords: magnetoencephalography (MEG), plaid, grating, visual evoked potential (VEP), oscillation 


\section{Abstract}

Electrophysiological recordings in primates indicate that visual gamma contains distinct broad- and narrow- band components that reflect different neuronal processes. Evidence suggests that cross-orientation masking of luminance-defined gratings should differentially modulate these two components. To test this we measured the effect of cross-orientation masking on the gamma response in twelve human participants using magentoencephalography (MEG).

Although both the amplitude and frequency of gamma were modulated by the presence of a cross-orientation mask, we failed to find evidence for distinguishable components: both broadband gamma at stimulus onset and sustained narrowband gamma were similarly modulated by mask contrast. However, we could not confirm the presence of masking effects due to mask contrast being confound with the contrast of the stimulus as a whole.

We therefore tested a further twelve participants in a second experiment in which the stimuli were: a plaid stimulus, the two component gratings which formed the plaid and the same two gratings but with Michelson contrast matched to the plaid. We found that gamma amplitude was reduced and gamma frequency increased to the plaid stimulus when compared to the contrastmatched gratings or to the sum of the two component gratings, indicating that visual gamma was indeed modulated by cross-orientation masking.

Surprisingly, we found that masking did not affect the pattern-onset evoked response, challenging previous hypotheses that cross-orientation suppression - the phenomenon by which the response to an oriented grating is suppressed by a cross-orientation mask - is driven by feedforward inputs to V1.

\section{Introduction}

The role of gamma oscillations in sensory processing remains an outstanding question within the field of neuroscience. Studies of the primate visual system have suggested the existence of two distinct gamma-band components within the local field potential (LFP) of V1. The first is a broadband response that extends to frequencies beyond $100 \mathrm{~Hz}$ (Ray \& Maunsell, 2011; Hermes et al., 2014) and is closely coupled to local firing rates (Jia et al., 2011; Ray \& Maunsell, 2011). The broadband nature of this response may reflect the spectral properties of excitatory post-synaptic potentials (EPSPs) within local pyramidal cells (Miller et al., 2009; Privman et al., 2013) or leakage of the spectral components of action potentials into the LFP (Zanos et al., 2011). The second is a narrowband response which appears to reflect the presence of an oscillation in the LFP that is coherent across an extended region of cortex (Jia et al., 2011) and is tuned differently to local firing rates (Gieselmann \& Thiele, 2008; Jia et al., 2011; Ray \& Maunsell, 2011). This narrowband response may reflect the influence of fast-spiking interneurons on the membrane potentials of pyramidal cells (Cardin et al., 2009).

In humans, there have been an extensive number of studies using MEG to measure responses in the gammaband to luminance-defined visual gratings and their tuning to stimulus parameters such as contrast (Hall et al., 2005; Perry et al., in press), size (Perry et al., 2013), orientation (Koelewijn et al., 2011), spatial frequency (Adjamian et al., 2004), motion (Swettenham et al., 2009) and eccentricity (Van Pelt \& Fries, 2013). Where direct comparisons have been possible, these findings have been in agreement with results from invasive electrophysiology in non-human primates (e.g. Hall et al., 2005).

Despite this, to our knowledge there have been no previous attempts to distinguish between broad- and narrow- band gamma in humans using MEG. This is perhaps not surprising: it is likely that population measures of broad- and narrow- band gamma covary for many stimulus manipulations, making the two responses potentially tricky to distinguish. Recently, however, Bartolo et al. (2011) have identified a stimulus manipulation that modulates population firing rates and narrowband gamma in opposite directions. During electrophysiological recordings in primate V1, they performed a comparison between luminance-defined gratings and plaids and found that, while population firing 
rates are greater for plaids than for gratings, the reverse is true for narrowband $(30-70 \mathrm{~Hz})$ gamma power.

This effect likely reflected the phenomenon of crossorientation suppression, a well-known effect in which the response of a cell to a grating of its preferred orientation is suppressed by the addition of a mask stimulus at a nonpreferred orientation (Morrone et al., 1982). Thus, in the Bartolo et al. (2011) study the addition of an orthogonally oriented component to a grating stimulus in order to form a plaid would have suppressed the responses of cells tuned to the orientation of the base grating, while increasing the responses of cells tuned to the orthogonal orientation. Thus, we can infer that for firing rates the increase in response to the mask orientation must exceed the suppression at the base orientation, leading to an elevated population-level response to the plaid stimulus, while for the gamma-band response the reverse must be the case (see also Lima et al., 2010).

Our aim in this study was to use this dissociation to test for the existence of separable components of the visual gamma response in humans. Our hypothesis was that the addition of an orthogonal mask orientation to a base grating (forming a plaid) should enhance the broadband gamma component (due to its expected coupling with firing rates) but suppress the narrowband component. We therefore used MEG to measure the effects of orthogonal masks on the amplitudes (as well as temporal parameters) of these two components. We were additionally interested to determine whether modulation of the gamma response by cross-orientation masks would provide any insight into the neurophysiological mechanisms of cross-orientation suppression.

\section{Materials and methods}

\section{Participants}

Twelve healthy volunteers (3 male; mean age: 22.7 yrs, range: 19-32 yrs) took part in Experiment 1, and a further twelve (4 female; mean age: 24.7 yrs, range: 21-27 yrs) took part in Experiment 2. All participants gave informed written consent. All had normal or corrected-to-normal vision. With one exception (noted below), each participant had a previously acquired structural MRI scan that was used for source localization. All procedures complied with the Code of Ethics of the World Medical Association (Declaration of Helsinki) and were approved by the ethics committee of the School of Psychology, Cardiff University.

\section{Stimuli and procedure}

In each experiment participants viewed a series of visually-presented gratings and plaids. All stimuli were created by combining vertical and/or horizontal luminance-defined square-wave gratings with a spatial frequency of 3 cycles/degree. Stimuli were masked by a square window measuring $8^{\circ} \times 8^{\circ}$ and presented centrally on a mean luminance $\left(26.5 \mathrm{~cd} / \mathrm{m}^{2}\right)$ grey background. All displays were generated by Matlab (The Mathworks, Inc: Natick, MA) using the Psychophysics Toolbox extensions (Brainard, 1997; Pelli, 1997; Kleiner et al., 2007), and presented on a gamma-corrected Mitsubishi Diamond Pro 2070 monitor $(1024 \times 768$ pixel resolution, $100 \mathrm{~Hz}$ refresh rate).

In Experiment 1, the four stimulus conditions were created by adding a horizontally-oriented mask grating at one of four Michelson contrasts - 0\%, 17\%, 33\% and $50 \%$ - to a vertically-oriented base grating at 50\% contrast. In Experiment 2 the five stimulus conditions were $50 \%$ contrast vertical, $100 \%$ contrast vertical, $50 \%$ contrast horizontal and $100 \%$ contrast horizontal gratings, along with a plaid stimulus formed from the addition of the $50 \%$ contrast horizontal and vertical gratings.

During each trial a centrally-presented red square $\left(\sim 0.2^{\circ}\right.$ in width) was present continuously and participants were instructed to maintain fixation on the square throughout. After a random interval between 1750-2000 ms the stimulus for that trial was presented for $1500 \mathrm{~ms}$ followed by a $1000 \mathrm{~ms}$ response period. In order to encourage participants to maintain attention to the stimuli, they were instructed to respond to stimulus offset from the screen by pressing a single button with the index finger of their right hand as rapidly as possible. If no response had been made within $750 \mathrm{~ms}$ of grating offset the fixation square was replaced by text reading 'Response not detected' for 250 
ms. In order to prevent button presses during the baseline period, participants were instructed to try to respond rapidly enough in every trial to prevent this text from appearing.

In experiment 1, participants viewed 60 trials per condition (240 trials in total), and in experiment 2 , they viewed 50 trials per condition (250 trials in total). All trials were presented in random order within an experiment.

\section{$M E G$ data acquisition and analysis}

Whole-head MEG recordings were made using a 275channel CTF radial gradiometer system sampled at 1200 Hz. An additional 29 reference channels were recorded for noise cancellation purposes, and the primary sensors were analysed as synthetic third-order gradiometers (Vrba \& Robinson, 2001). Three of the 275 channels were turned off due to excessive sensor noise.

To achieve MRI/MEG co-registration, fiduciary markers were placed at fixed distances from three anatomical landmarks (nasion and pre-auricular) identifiable in the participants' anatomical MRIs. Fiduciary locations were verified afterwards using high-resolution digital photographs.

Data were recorded in $4 \mathrm{~s}$ epochs beginning at $1.75 \mathrm{~s}$ before stimulus onset. Artefact rejection was performed offline by manually inspecting the data and discarding trials with excessive muscle or head-movement-related artefacts.

All participants (except one) had a previously acquired anatomical MRI scan at $1 \mathrm{~mm}$ isotropic voxel resolution. For source localisation, a multiple local spheres forward model (Huang et al., 1999) was derived by fitting spheres to the individual's brain surface extracted from their MRI using FSL's Brain Extraction Tool (Smith, 2002). For one participant in Experiment 2, no MRI data was available, so we compared the relative locations of that participant's fiducials with those in all other participants and used the brain surface from the participant with the closest matching fiducial locations.
For analysis of the gamma response, each data set was bandpass filtered using a 4th order bi-directional IIR Butterworth filter at $30-70 \mathrm{~Hz}$ (this choice was based on the frequency range of visual gamma found across individuals in previous studies; e.g. Muthukumaraswamy et al., 2010). The synthetic aperture magnetometry (SAM) method (Robinson \& Vrba, 1999) was then used to create a set of beamformer weights for the whole brain at $4 \mathrm{~mm}$ isotropic voxel resolution for each participant. Data covariance matrices used to create the weights were calculated from the concatenation of all trials regardless of condition (but excluding trials containing artefacts), so that a common set of weights was used across conditions. Virtual sensors were constructed at each voxel, and paired- $t$ statistical images of source power (Student's $t$ statistic) for the $1.5 \mathrm{~s}$ period of stimulus presentation contrasted with a baseline period (the $1.5 \mathrm{~s}$ period prior to stimulus onset) were generated for each participant. Source images were calculated from all trials regardless of condition in order to maximise the signal to noise ratio of the resulting statistical images (under the assumption that, for each participant, the location of the strongest gamma source would not differ between conditions).

In order to perform a virtual sensor analysis of the gamma response across conditions, the individual paired- $t$ SAM images of each participant were examined and the location of intensity peaks in each image were obtained. Although we would expect the presence of bilateral activation - due to visual stimulation being present in both visual hemifields - in some participants the beamformer images lacked the spatial resolution to distinguish two distinct peaks across the hemispheres of occipital cortex. In order to maintain consistency across participants we therefore restricted the virtual sensor analysis to the location of the single largest $t$-staristcal value within the occipital cortex regardless of hemisphere.

Virtual sensor timeseries were generated for each condition per participant by using the SAM beamformer method a second time, this time for this single obtained location per individual (again a common set of weights was used across conditions by calculating data covariance across all trials except those containing artefacts). Timefrequency analysis was then performed on the resulting 
timeseries using the Hilbert transform from 4 to $120 \mathrm{~Hz}$ in $0.5 \mathrm{~Hz}$ steps (using a bandpass 3rd order Butterworth filter with $8 \mathrm{~Hz}$ bandwidth). Response magnitude in the resulting spectrograms was calculated as percentage change in amplitude relative to baseline.

For analyses of the pattern-onset event-related field (ERF), we followed the method proposed by Robinson (2004). Beamfomer weights were calculated from unaveraged data bandpass filtered at $1-30 \mathrm{~Hz}$. Evoked timeseries were then calculated by averaging the filtered data across trials and baseline correcting against the $150 \mathrm{~ms}$ prior to stimulus onset. SAM images were then produced by projecting the evoked timeseries through the beamformer weights and calculating power within a window from 70-110ms after stimulus onset at each voxel. These measures of power were normalised using the voxelwise standard error across trials, which was estimated using a jackknife procedure. 70-110ms was chosen as the time window which contained the first prominent peak in the sensor-level evoked timeseries across all participants. As in the gamma analysis, measures of source power were calculated across all trials regardless of condition.

Peak activity was found for each participant in these new images, and virtual sensor timeseries were generated for each condition at this location using beamformer weights which were pseudo-Z corrected with respect to projected sensor noise (Robinson \& Vrba, 1999). ERF timeseries for each condition were then calculated by averaging across trials and baseline correcting (again against the $150 \mathrm{~ms}$ prior to stimulus onset) for each participant.

\section{Results}

\section{Experiment 1}

In Experiment 1 a vertical test stimulus was presented at $50 \%$ contrast, accompanied by a horizontal mask stimulus in one of four contrast conditions: $0 \%, 17 \%, 33 \% \& 50 \%$. The aim was to determine if cross-orientation masking would differentially modulate broad- and narrow- band components of the gamma response. In particular, MEGrecorded gamma in humans has a consistent morphology across both

individuals and recording sessions (Muthukumaraswamy et al., 2010): an initial transitory 'spike' in gamma-band amplitude which has a broad spectral profile and (like single cell firing rates) rises and falls rapidly after stimulus onset, and a sustained response which has narrow spectral profile and appears to persist for as long as the stimulus is present. Our hypothesis was that these might respectively correspond to the broad- and narrowband responses found in invasive neurophysiology, and

\begin{tabular}{|c|ccc|ccc|}
\hline \multicolumn{1}{c|}{} & \multicolumn{3}{|c|}{ Gamma } & \multicolumn{3}{c|}{ ERF } \\
\hline Participant & $x(\mathrm{~mm})$ & $y(\mathrm{~mm})$ & $\mathrm{z}(\mathrm{mm})$ & $x(\mathrm{~mm})$ & $y(\mathrm{~mm})$ & $\mathrm{z}(\mathrm{mm})$ \\
\hline 1 & 1 & -108 & -9 & -26 & -99 & -7 \\
2 & -4 & -102 & 2 & 1 & -94 & -32 \\
3 & -16 & -109 & -2 & -2 & -103 & -25 \\
5 & -18 & -101 & -5 & -10 & -106 & -9 \\
6 & -11 & -97 & 15 & 21 & -83 & 9 \\
7 & -6 & -93 & 12 & -9 & -101 & 27 \\
8 & -20 & -100 & 2 & -20 & -101 & -3 \\
9 & -12 & -105 & 11 & -20 & -103 & -4 \\
10 & -18 & -93 & -2 & -11 & -98 & 24 \\
11 & 3 & -101 & -8 & -10 & -109 & 1 \\
12 & -20 & -96 & 6 & -29 & -96 & 11 \\
\hline Mean & -21 & -103 & 11 & -7 & -102 & 15 \\
\hline Standard deviation & -12 & -101 & 3 & -10 & -100 & 1 \\
\hline
\end{tabular}

Table 1: Talairach coordinates of virtual sensor locations for both gamma and ERF analyses in Experiment 2. 
5 The effects of cross-orientation masking on gamma
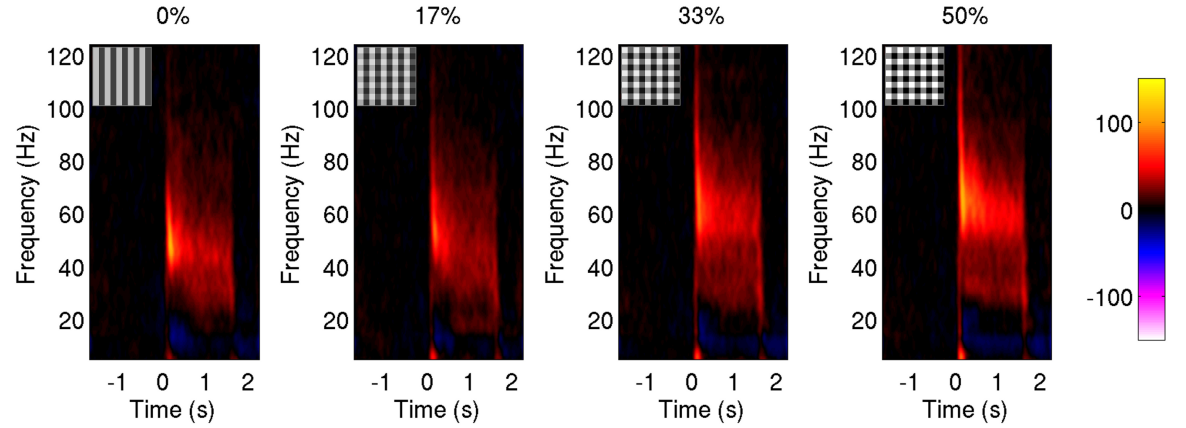

Figure 1: Spectrograms of the virtual sensor responses relative to stimulus onset in each of the four conditions of mask contrast in Experiment 1. Insets illustrate the stimulus type used in each condition. The colour scale represents amplitude as percentage change from baseline.

we specifically tested for differences in the modulation of these two components by mask contrast.

SAM images of source power averaged across all trials were created for each participant. A virtual sensor analysis was performed at the location of peak activity separately for each participant (Talairach coordinates of peaks are given in Table 1; the group average peak location corresponded to the left posterior cuneus around the approximate border of Brodmann areas 17 and 18), and spectrograms of the response at that location were calculated for each condition (Figure 1). Each spectrogram was then averaged across time in the interval $400-1500 \mathrm{~ms}$ in order to produce amplitude spectra of the sustained part of the visual gamma response (Figure 2 upper panel). These spectra revealed a clear modulation of visual gamma by the masking stimulus, with the spectral peak being initially suppressed as mask contrast increased from $0 \%$ to $17 \%$ but then increasing again at a higher frequency as mask contrast increased further.

We parameterised each spectrum by finding the amplitude
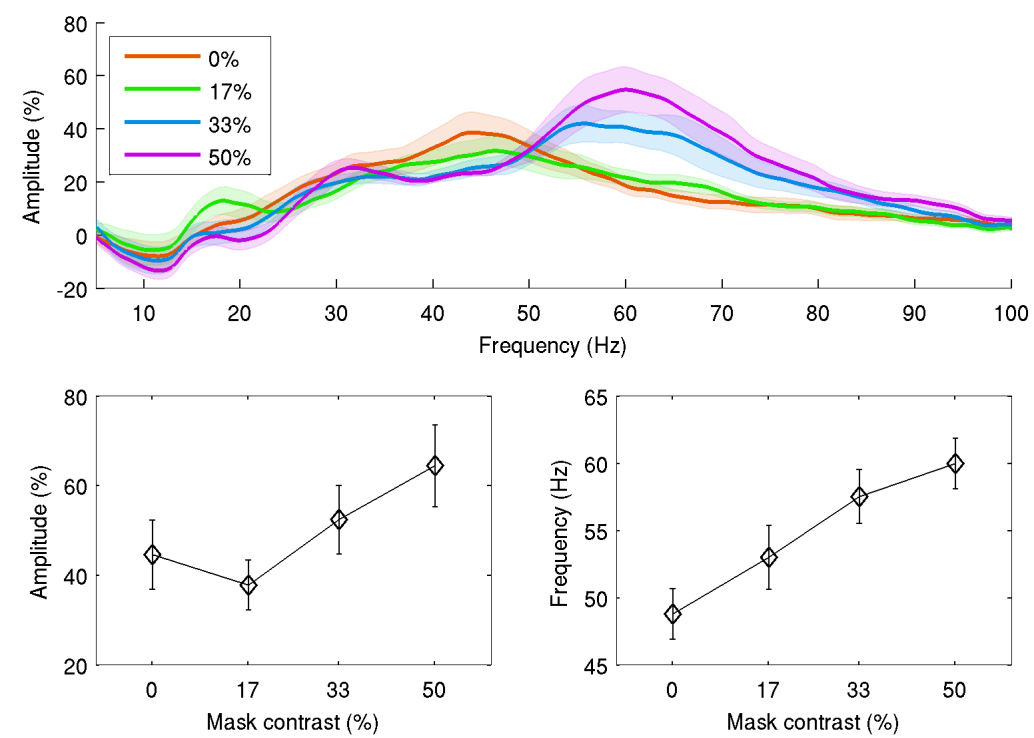

Figure 2: Plots of data from the sustained gamma component (400-1500 ms) in Experiment 1. Upper panel displays group mean (+/- SE) amplitude spectra for each of the four conditions of mask contrast. Lower panels display group mean (+/SE) peak amplitude (left panel) and frequency (right panel) of sustained gamma against mask contrast. 

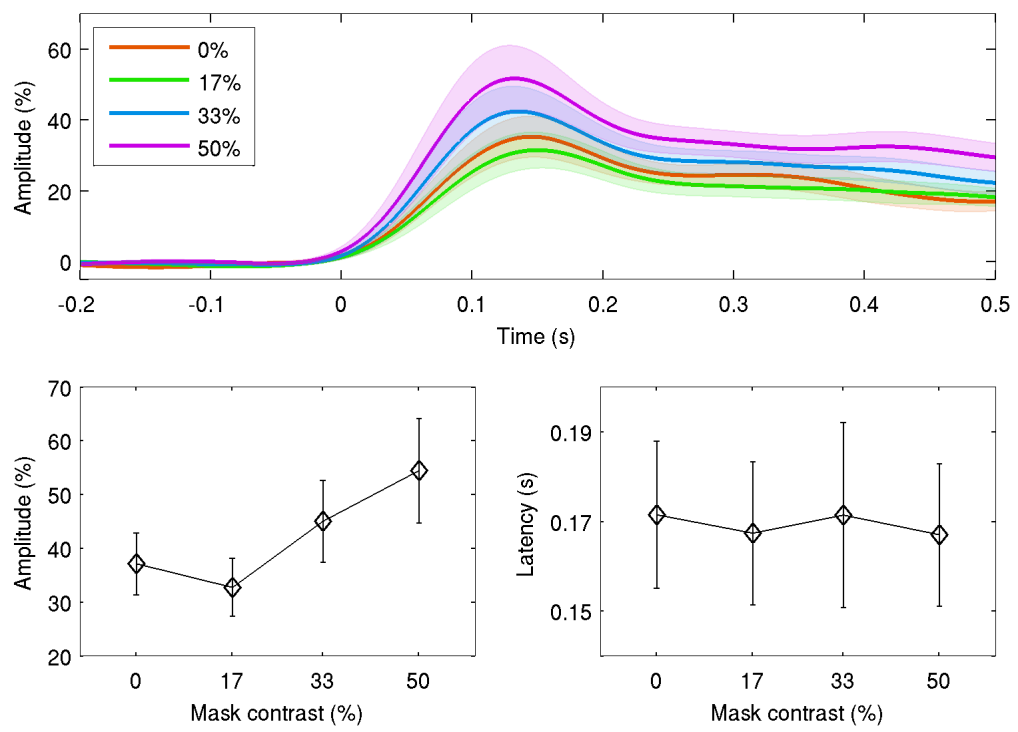

Figure 3: Plots of data from the broadband gamma component $(30-120 \mathrm{~Hz})$ in Experiment 1. Upper panel displays group mean (+/- SE) broadband amplitude against time (relative to stimulus onset) for each of the four conditions of mask contrast. Lower panels display group mean (+/- SE) peak amplitude (left panel) and latency (right panel) of broadband gamma against mask contrast.

and frequency of the spectral peak in the $40-70 \mathrm{~Hz}$ range (Figure 2 lower panels; the high-pass of the filter was raised to $40 \mathrm{~Hz}$ from $30 \mathrm{~Hz}$ in order to exclude the effects of spectral peaks in the beta-band which were present in some participants), and tested for differences between conditions using one-way repeated-measures ANOVAs with Greenhouse-Geisser correction for deviations from sphericity. This data confirmed that both the amplitude $(F(1.5,16.7)=6.78, p=0.01)$ and frequency $(F(2.0,22.1)$ $=9.96, p=0.001)$ of the visual gamma response were significantly modulated by mask contrast. In the case of amplitude the pattern of modulation was consistent with that seen in the full spectra: an initially decreasing amplitude as the mask was introduced at $17 \%$ contrast gave way to a sharply increasing amplitude at higher contrasts. In the case of frequency, the group average spectra shown in the upper panel of Figure 2 gives the impression of a sharp shift in gamma frequency between $17 \%$ and $33 \%$ contrast. However a plot of the groupaveraged peak frequencies reveals that the gamma frequency in fact appeared to be a smoothly increasing function of mask contrast (lower right panel of Figure 2), with group mean frequency increasing from $49 \mathrm{~Hz}$ in the $0 \%$ condition to $60 \mathrm{~Hz}$ in the $50 \%$ condition.
To analyse the transient gamma spike around stimulus onset we averaged each spectrogram across frequency in the range $30-120 \mathrm{~Hz}$ to produce timeseries of broadband gamma power (Figure 3 upper panel). As with the gamma spectra, these timeseries were parametrised, this time with respect to the amplitude and latency of the peak response occuring between 50-350ms (Figure 3 lower panels). Amplitude $(F(1.2,12.7)=8.39, p=0.01)$, but not latency $(F(2.4,26.8)=0.11, p=0.9)$, was significantly modulated by the contrast of the mask stimulus. The pattern of amplitude modulation was virtually identical to that shown for the sustained gamma (compare lower left panels of Figures $2 \& 3$ ), suggesting that the amplitude of the transient and sustained gamma responses were not differentially modulated by mask contrast.

If the sustained gamma and the transient 'spike' were differentially modulated by the mask stimulus, we would expect the difference in amplitude between the two measures to vary between conditions. In fact, we found no significant difference between the conditions for this measure $(F(2.6,28.3)=0.44, p=0.7)$.

One further possibility that we considered is that the 

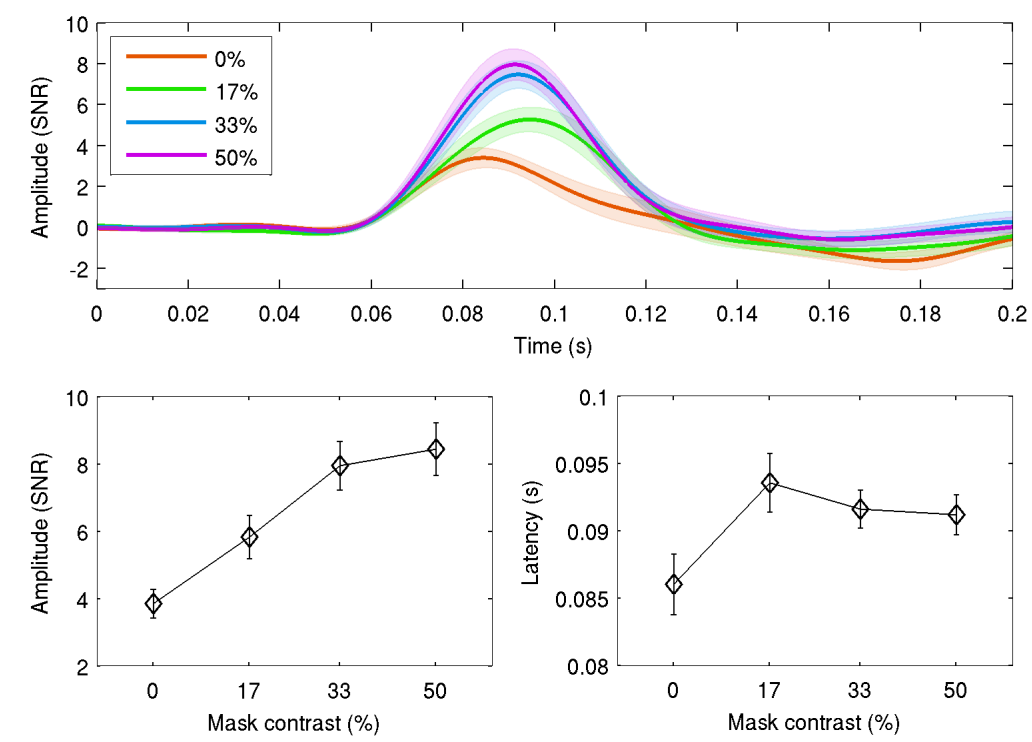

Figure 4: Plots of data from the pattern-onset ERF in Experiment 1. Upper panel displays group mean (+/- SE) evoked amplitude against time (relative to stimulus onset) for each of the four conditions of mask contrast. Lower panels display group mean (+/-SE) peak amplitude (left panel) and latency (right panel) of the ERF.

gamma 'spike' is formed from the combination of the sustained response and a weaker broadband response. Thus, we subtracted the amplitude spectrum of the sustained response from the spectrogram for each condition for each participant and re-analysed the transitory response in the residual time-frequency data. While the differences in amplitude between conditions became smaller, the rank order of condition means remained the same and the difference between conditions remained significant $(F(1.3,14.2)=4.3, p=0.049)$.

Thus, we did not find evidence for an additional broadband component of the gamma 'spike' which was differentially modulated by mask contrast relative to the sustained gamma. Indeed the amplitudes of the transient and sustained gamma were strongly correlated across participants within each condition $(0 \%$ condition: $r=$ $0.84, p=7 \mathrm{e}-4 ; 17 \%: r=0.83, p=0.001 ; 33 \%: r=0.89, p$ $=1 \mathrm{e}-4 ; 50 \%: r=0.88, p=1 \mathrm{e}-4$; Pearson's correlation coefficient) lending further weight to the conclusion that the two measures, far from being independent, were in fact closely coupled.

Grating stimuli are also known to evoke event-related fields (ERFs) at pattern-onset, and so we additionally analysed these ERFs to determine if they too were modulated by the contrast of mask stimuli in a manner consistent with that found for the gamma response. To carry out this analysis we generated SAM images of evoked source power and performed a virtual sensor analysis at the location of peak activity (Talairach coordinates of peaks are given in Table 1; as before the group average peak location corresponded to the left posterior cuneus around the approximate border of Brodmann areas 17 and 18). For each participant we then averaged the virtual sensor timeseries across trials within a condition in order to calculate the ERF timeseries (Figure 4 upper panel).

As with the transient gamma, we parameterised these ERFs by the amplitude and latency of the first prominent peak in each evoked timeseries (Figure 4 lower panels). Based on the range of latencies of this response across conditions and participants (75-105 ms) this peak most likely corresponded to the magentoencephalographic equivalent of the $\mathrm{C} 1$ visual evoked potential (VEP) (Odom et al., 2004). Both the amplitude $(F(2.0,22.2)=$ $69.9, p=3 \mathrm{e}-10)$ and latency $(F(2.0,22.3)=20.07, p=1 \mathrm{e}-$ $5)$ of the pattern-onset ERF were significantly modulated by mask contrast. The amplitude of the ERF did not show 


\begin{tabular}{|c|ccc|ccc|}
\cline { 2 - 7 } \multicolumn{1}{c|}{} & \multicolumn{3}{c|}{ Gamma } & \multicolumn{3}{c|}{ ERF } \\
\hline Participant & $x(\mathrm{~mm})$ & $y(\mathrm{~mm})$ & $\mathrm{z}(\mathrm{mm})$ & $x(\mathrm{~mm})$ & $y(\mathrm{~mm})$ & $\mathrm{z}(\mathrm{mm})$ \\
\hline 1 & -15 & -104 & 8 & -26 & -99 & -7 \\
2 & 30 & -90 & 24 & 1 & -94 & -32 \\
4 & 0 & -99 & -13 & -2 & -103 & -25 \\
5 & 28 & -97 & 0 & -10 & -106 & -9 \\
6 & -10 & -94 & 7 & 21 & -83 & 9 \\
7 & 5 & -103 & -5 & -9 & -101 & 27 \\
8 & -16 & -97 & -2 & -20 & -101 & -3 \\
9 & -5 & -100 & 10 & -20 & -103 & -4 \\
10 & 0 & -98 & -21 & -11 & -98 & 24 \\
11 & -14 & -94 & -4 & -10 & -109 & 1 \\
12 & -1 & -99 & 27 & -29 & -96 & 11 \\
\hline Mean & -5 & -71 & 13 & -7 & -102 & 15 \\
\hline Standard deviation & 0 & -96 & 4 & -10 & -100 & 1 \\
\hline
\end{tabular}

Table 2: Talairach coordinates of virtual sensor locations for both gamma and ERF analyses in Experiment 2.

any evidence of suppression by the mask and instead was monotonically increasing with mask contrast, in distinction to the data for the gamma response. The latency of the ERF was delayed in conditions in which the mask was present (confirmed by Bonferroni-corrected post-hoc $t$-tests which found significant pairwise differences between the $0 \%$ contrast and each of the other three conditions, but not between any other pairwise comparisons).

\section{Experiment 2}

The findings of Experiment 1 appear difficult to reconcile with the results of Bartolo et al. (2011). Where they found that the power of the gamma-band was substantially reduced for plaids versus gratings, we found the reverse result (with exception of the $17 \%$ mask condition for which gamma amplitude was slightly reduced compared to the grating condition). However, one key difference between these studies is that Bartolo et al. compared gratings and plaids matched for Michelson contrast, whereas in Experiment 1 the Michelson contrast of the stimulus increased with mask contrast, producing a potential confound in the data. Given that previous MEG studies have shown that gamma amplitude is an increasing function of Michelson contrast for grating stimuli (Hall et al., 2005; Perry et al., in press), we might expect that the increasing gamma amplitude to the mask stimulus might exceed the reduction of the gamma response due to cross-orientation masking.

Likewise, gamma frequency has been found to increase with Michelson contrast for grating stimuli (Perry et al., in press). Thus, the effects on gamma amplitude and frequency found in Experiment 1 may have been dominated by changes in luminance contrast between conditions and may not have reflected the effects of crossorientation masking.

Thus, we ran a second experiment, to determine whether the gamma response to plaid stimuli is determined by the Michelson contrast alone. The procedure was identical to that used for Experiment 1, but our test stimuli were as follows: a horizontal 50\% contrast grating, a vertical 50\% grating, a plaid stimulus created by combiming the two $50 \%$ gratings, a horizontal $100 \%$ contrast grating and a vertical $100 \%$ grating. This allowed us to compare the response to the plaid with the response to the two component stimuli from which it was formed, as well as with two gratings with Michelson contrast matched to that of the plaid.

With the new data, we repeated the SAM and virtual sensor analyses for the gamma response as outlined for 
9 The effects of cross-orientation masking on gamma
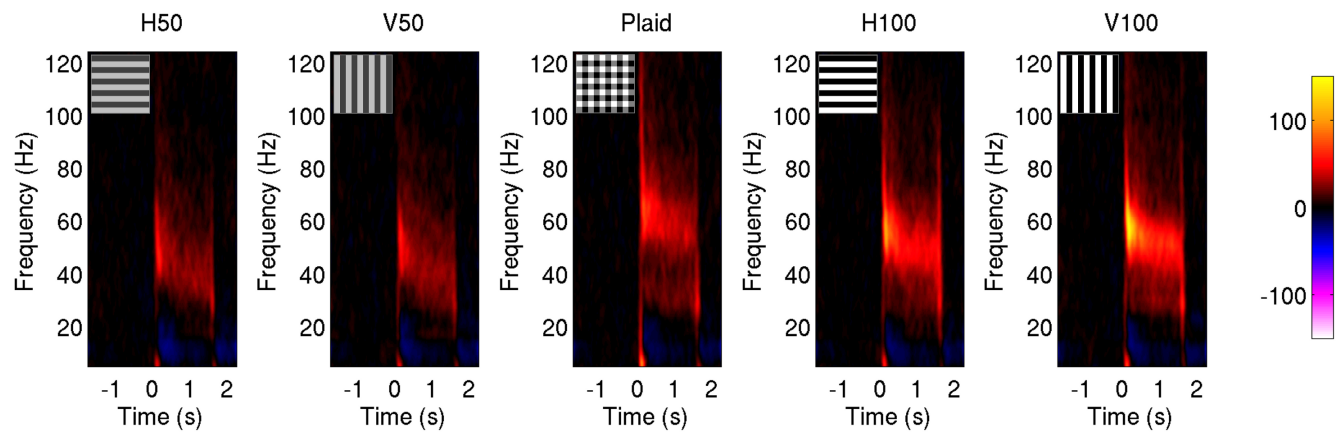

Figure 5: Spectrograms of the virtual sensor responses relative to stimulus onset in each of the five conditions in Experiment 2 (H50: horizontal grating, 50\% contrast; V50: vertical grating, 50\% contrast; Plaid; H100: horizontal grating, 100\% contrast; V100: vertical grating, 100\% contrast). Insets illustrate the stimulus type used in each condition. The colour scale represents amplitude as percentage change from baseline.

Experiment 1 above (Talairach coordinates of peaks are given in Table 2; the group average peak location corresponded to the medial surface of the left cuneus around the approximate border of Brodmann areas 17 and 18; group average spectrograms by condition are shown in Figure 5). The spectra of the sustained gamma response were again parameterised by the amplitude and frequency of the spectral peak within the $40-70 \mathrm{~Hz}$ range and tested for significant differences as in Experiment 1 (Figure 6).
Both gamma amplitude $(F(3.1,33.2)=21.38, p=7 \mathrm{e}-8)$ and frequency $(F(2.3,25.0)=19.99, p=4 \mathrm{e}-6)$ were modulated by stimulus condition. As a result we performed a series of planned comparisons between specific conditions of interest using paired $t$-tests.

Increasing stimulus contrast from $50 \%$ to $100 \%$ increased the amplitude of the gamma response for both horizontal $(t(11)=5.9, p=1 \mathrm{e}-4)$ and vertical $(t(11)=7.0, p=2 \mathrm{e}-5)$
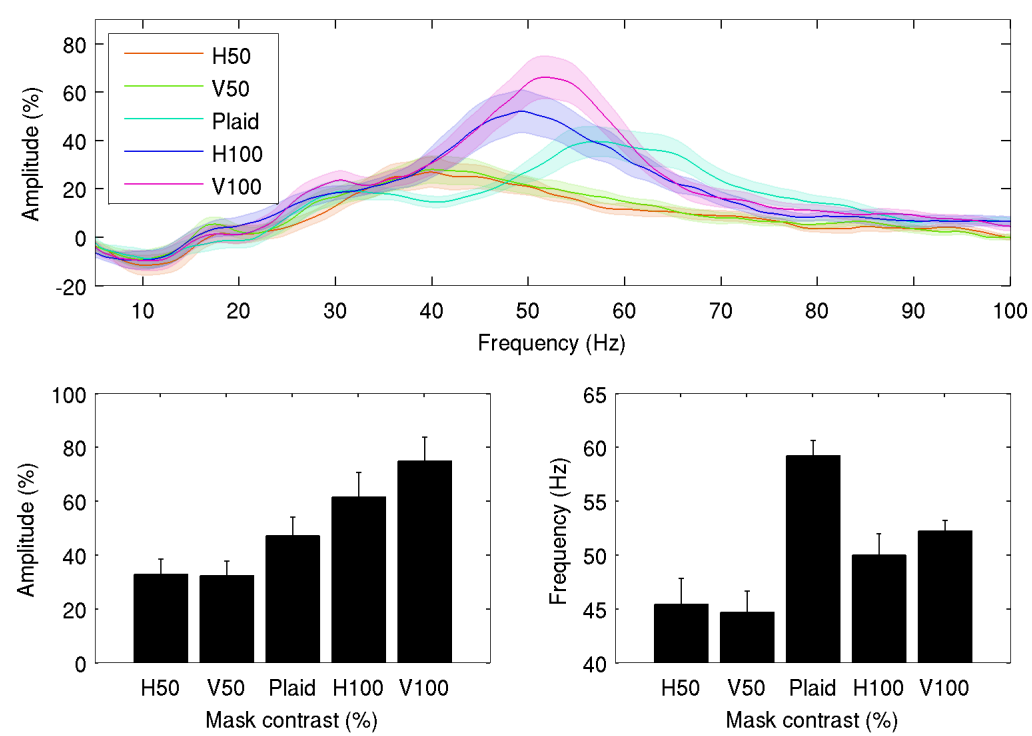

Figure 6: Plots of data from the sustained gamma component (400-1500 ms) in Experiment 2. Upper panel displays group mean (+/- SE) amplitude spectra for each of the five stimulus conditions (H50: horizontal grating, 50\% contrast; V50: vertical grating, 50\% contrast; Plaid; H100: horizontal grating, 100\% contrast; V100: vertical grating, 100\% contrast). Lower panels display group mean (+/- SE) peak amplitude (left panel) and frequency (right panel) of sustained gamma against mask contrast. 

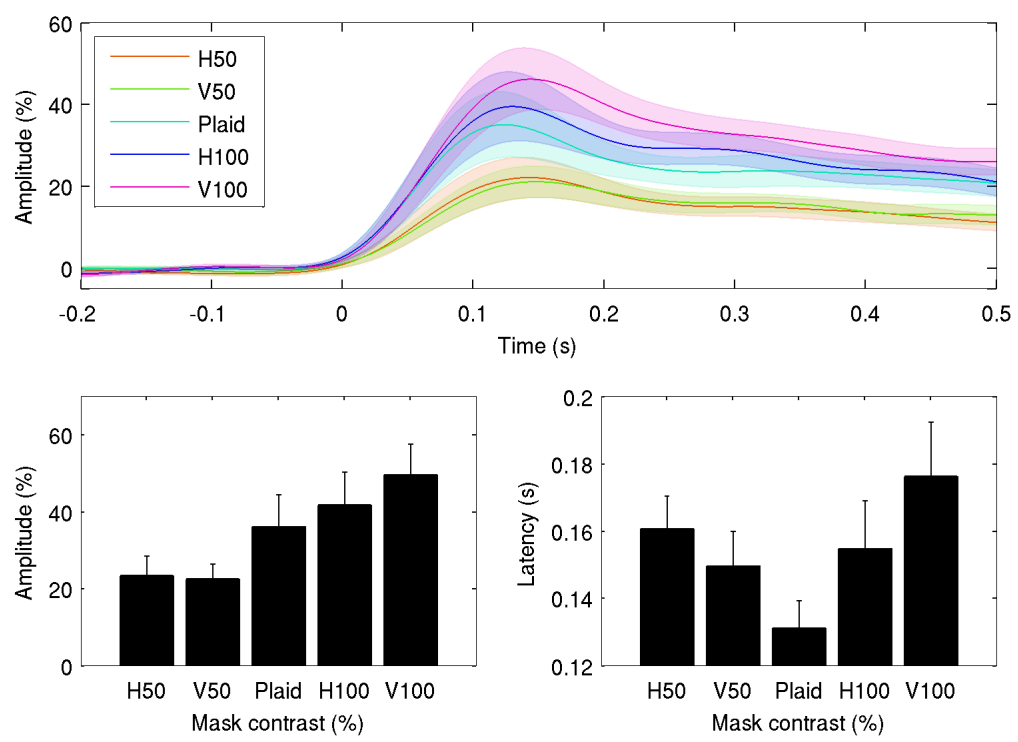

Figure 7: Plots of data from the broadband gamma component $(30-120 \mathrm{~Hz})$ in Experiment 2. Upper panel displays group mean (+/- SE) broadband amplitude against time (relative to stimulus onset) for each of the five stimulus conditions (H50: horizontal grating, 50\% contrast; V50: vertical grating, 50\% contrast; Plaid; H100: horizontal grating, 100\% contrast; V100: vertical grating, 100\% contrast). Lower panels display group mean (+/- SE) peak amplitude (left panel) and latency (right panel) of broadband gamma against mask contrast.

gratings, consistent with previous MEG findings in humans (Hall et al., 2005; Perry et al., in press). When averaged across orientations, a doubling of the stimulus contrast stimulus increased the gamma amplitude by a factor of 2.3 , indicating that the response to a $100 \%$ contrast grating was approximately the sum of two $50 \%$ gratings, and suggesting that the effect of stimulus contrast was approximately additive within an orientation. Despite being matched for Michelson contrast, the gamma amplitude to the plaid was significantly less than the mean of the response to the two $100 \%$ gratings $(t(11)=$ 6.88, $p=3 \mathrm{e}-5)$ and was also less than the sum of the amplitudes to the two $50 \%$ gratings, although this difference did not quite reach significance $(t(11)=1.97, p$ $=0.07$ ). Thus, the data provided evidence that the gamma amplitude to a plaid stimulus is reduced relative to that produced by gratings when matched for Michelson contrast.

Increasing stimulus contrast from $50 \%$ to $100 \%$ also increased the frequency of the gamma response for both horizontal $(t(11)=2.8, p=0.02)$ and vertical $(t(11)=3.5$, $p=0.005)$ gratings. Again this was in agreement with previous research (Perry et al., in press). Averaged across orientations the mean frequency increased from $46 \mathrm{~Hz}$ at $50 \%$ contrast to $52 \mathrm{~Hz}$ at $100 \%$ contrast. The mean gamma frequency to the plaid was $59 \mathrm{~Hz}$, which was significantly greater than the mean of the response to the $100 \%$ stimuli $(t(11)=5.7, p=1 \mathrm{e}-4)$. Thus, the addition of an orthogonal mask grating at $50 \%$ contrast increased the gamma frequency by more than double the increase that occurred when adding $50 \%$ contrast to the base grating. This confirmed that the increase in gamma frequency with increasing mask contrast seen in Experiment 1 was greater than would be expected simply from the confounding effects of increased contrast of the stimulus.

We additionally analysed the transient gamma 'spike' (Figure 7), using the same approach as used in Experiment 1. Again we found that the amplitude of the response was significantly different between conditions $(F(2.4,26.3)=12.6, p=7 \mathrm{e}-5)$ but followed a highly similar pattern to that found for the sustained gamma amplitude (compare the lower left panels of Figures 6 \& 

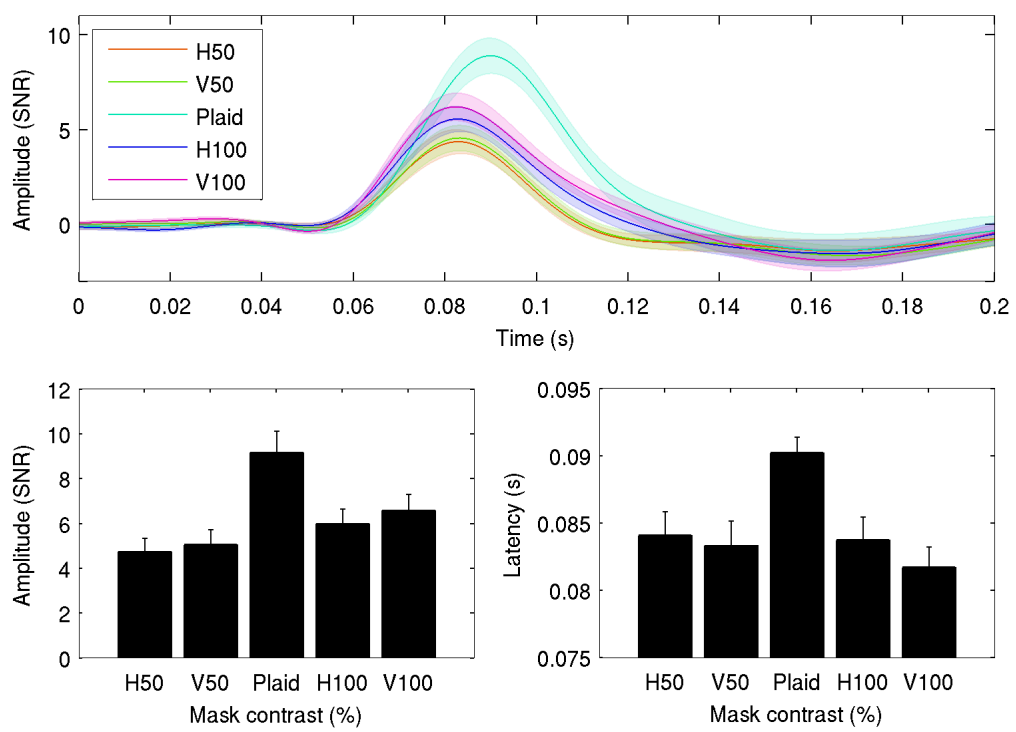

Figure 8: Plots of data from the pattern-onset ERF in Experiment 2. Upper panel displays group mean (+/- SE) evoked amplitude against time (relative to stimulus onset) for each of the five stimulus conditions (H50: horizontal grating, 50\% contrast; V50: vertical grating, 50\% contrast; Plaid; H100: horizontal grating, $100 \%$ contrast; V100: vertical grating, $100 \%$ contrast). Lower panels display group mean (+/-SE) peak amplitude (left panel) and latency (right panel) of the ERF.

7). As before, the amplitudes of the sustained and transient gamma were strongly correlated across participants in each condition (horizontal 50\%: $r=0.76, p$ $=0.004$; vertical 50\%: $r=0.67, p=0.01$; plaid: $r=0.76$, $p=0.004$; horizontal 100\%: $r=0.74, p=0.006$; vertical 100\%: $r=0.68, p=0.02$; Pearson's correlation coefficient). Thus, we did not find clear evidence of a dissociation between the transient and sustained gamma responses in Experiment 2. Despite a trend for plaid stimuli to produce an earlier gamma spike, we did not find a significant difference in peak latency between conditions $(F(2.5,27.1)=2.3, p=0.11)$.

As in Experiment 1, we were also interested in comparing our results from the gamma response with data from the pattern-onset ERF. We repeated the SAM and virtual sensor (Talairach coordinates of peaks are given in Table 2 ; as before the group average peak location corresponded to the medial surface of the left cuneus around the approximate border of Brodmann areas 17 and 18) analyses on the data from Experiment 2 (Figure 8) and again parameterised the ERFs by the amplitude and latency of the response peak. Both ERF amplitude
$(F(2.4,26.4)=39.49, p=3 \mathrm{e}-9)$ and latency $(F(2.5,27.3)=$ $39.31, p=2 \mathrm{e}-9)$ were significantly modulated by stimulus condition.

Increasing stimulus contrast from $50 \%$ to $100 \%$ significantly increased the amplitude for both horizontal $(t(11)=6.0, p=9 \mathrm{e}-5)$ and vertical $(t(11)=6.1, p=8 \mathrm{e}-5)$ stimuli. However, averaged across orientations, the increase in amplitude from $50 \%$ to $100 \%$ contrast was only around a third of the magnitude of the response to the 50\% stimuli, suggesting that ERF amplitude was subject to response saturation as contrast increased towards $100 \%$. Conversely, the amplitude of the ERF to the plaid was only slightly less (by around 5\%) than the sum of the amplitudes to the two 50\% stimuli, and therefore was significantly larger than the mean amplitude of the two $100 \%$ contrast stimuli $(t(11)=6.5, p=4 \mathrm{e}-5)$ but not significantly different from the sum of the amplitudes to the $50 \%$ contrast stimuli $(t(11)=1.1, p=$ 0.3). Thus, ERF amplitude appeared to saturate when contrast increased within an orientation, but was approximately additive between orientations, suggesting that with respect to the amplitude of the pattern-onset 
ERF the two orientations only weakly interacted at best. Increasing stimulus contrast from $50 \%$ to $100 \%$ produced a slight increase in ERF latency, but this was significant only for vertical $(t(11)=2.4, p=0.04)$ and not horizontal $(t(11)=0.73, p=0.48)$ gratings. Conversely, ERF peak latency was significantly slowed to the plaid stimulus relative to the mean across orientations of both the $100 \%$ $(t(11)=12.6, p=7 \mathrm{e}-18)$ and $50 \%(t(11)=7.1, p=2 \mathrm{e}-5)$ contrast gratings. Thus the evidence of Experiment 2 suggested that cross-orientation interactions affect the latency, but not the amplitude, of the pattern-onset ERF.

\section{Discussion}

Electrophysiology in non-humans primates has provided evidence that the gamma response in primary visual cortex contains at least two distinct sub-components: a broadband response that is closely-coupled to local firings rates and a narrowband response which reflects a coherent oscillation in the LFP across an extended region of cortex which is tuned differently to local firing rates (Jia et al., 2011; Ray \& Maunsell, 2011). Here, we investigated whether similar sub-components of the gamma response could be found in human visual cortex using MEG. Based on the findings of Bartolo et al. (2011) that population firing rates were enhanced to plaid versus grating stimuli, but that the amplitude of the narrowband gamma response showed the reverse effect, we tested for the presence of a similar difference in sub-components the gamma response in humans. Our specific hypothesis was that in our data the transient broadband gamma spike would follow the pattern of responding seen for firing rates while the sustained gamma would show the pattern seen in Bartolo et al. for the gamma response. This was tested by introducing an orthogonal mask orientation to a base grating at progressively increasing mask contrast (Experiment 1) and by comparing the response to a plaid stimulus relative to gratings whose Michelson contrast matched either the individual components of the plaid or the plaid stimulus as a whole (Experiment 2).
Absence of evidence for distinguishable gamma subcomponents

Across both experiments we failed to find evidence to support the above hypothesis or the more general idea that there are dissociable sub-components of the gamma response. In Experiment 1, we found that the amplitude of transitory and sustained gamma responses were both similarly modulated by the contrast of the mask stimulus, and were strongly correlated across participants. We were able to show that this effect remained when the spectral profile of the sustained response was subtracted from the transitory response, indicating that this lack of difference was not due to contamination of the broadband transitory gamma by the narrowband sustained response. Our results from Experiment 2 demonstrated that both the transitory and sustained gamma showed a similar pattern of response (reduced for plaids relative to gratings matched for contrast) to that shown by Bartolo et al. (2011) for the gamma response (see also Lima et al., 2010). Thus, we did not find evidence in humans for the broadband gamma response coupled to firing rates that has been reported in non-human primates (Jia et al., 2011; Ray \& Maunsell, 2011; Hermes et al., 2014). Instead, our evidence is consistent with the conclusion that the gamma response measured in humans primarily corresponds to the narrow-band response found in non-human primates.

The presence of a broadband response in the gamma frequency range to luminance-defined gratings has been demonstrated a number of times in LFP recording in nonhuman primates (Jia et al., 2011; Ray \& Maunsell, 2011; Hermes et al., 2014). This raises the question as to why we were unable to find any clear evidence for this response in this study. One possibility we considered is that the broadband response might have been most evident at frequencies above $120 \mathrm{~Hz}$ and hence by only considering lower frequencies we missed the true effect. However, when our data were re-analysed at higher frequencies we found that gamma power tended to drop off rapidly for most participants as frequency increased above $120 \mathrm{~Hz}$, despite the broadband response clearly extending to much higher frequencies in studies using invasive electophysiology (Ray \& Maunsell, 2011; Hermes et al., 2014). Thus, we did not find any evidence 
to support the idea that we missed experimental effects occuring at higher frequencies.

An alternative explanation for our failure to find the predicted effect for broadband gamma is that, while we have assumed that the findings of Bartolo et al. (2011) with respect to firing rates should generalise to the broadband response based on previous evidence that the amplitude of broadband gamma is coupled to local firing rates at electrophysiological recording sites (Jia et al., 2011; Ray \& Maunsell, 2011), we are not aware that this has been demonstrated experimentally. If the broadband response becomes decoupled from local firing rates in the presence of cross-orientation masking, then this could lead to the response being modulated differently from firing rates in response to plaid stimuli. In this case our underlying assumption that the comparison between grating and plaid stimuli would reveal differences in gamma sub-components would be invalid, and the experiments used here would not be capable of distinguishing between these sub-components. Only direct tests of the relative amplitude of broadband gamma to plaid and grating stimuli with invasive recordings can determine if this is the case.

A further possible explanation is that the amplitude of the broadband response did match population firing rates in our paradigm, but that this sub-component of gamma is simply not measurable non-invasively using MEG. Indeed, in order for electrophysiological activity to generate a measurable magnetic field outside the head, it is necessary for the activity to be coherent over extended regions of cortex, such that the generated fields sum constructively. However, evidence from non-human primates implies that broadband gamma is only weakly coherent across nearby recording sites (Jia et al., 2011). Thus the broadband response may not have sufficient coherence to produce an externally measurable signal, and for this reason may not be measurable by MEG.

Effects of cross-orientation masking on the gamma response

In Experiment 1 we found clear evidence that both the amplitude and frequency of the gamma response are modulated by the presence (and contrast of) an orthogonal mask. However, previous MEG studies (Hall et al., 2005; Perry et al., in press) have demonstrated that both gamma amplitude and frequency to luminance-defined gratings are linearly increasing with Michelson contrast. Thus our results in that experiment were confounded by differences in contrast of the stimuli as a whole across conditions, making it difficult to unambiguously determine the effects of cross-orientation masking on the gamma response. This left the possibility that no masking effects were present and that responses to plaids merely reflected the summation of responses to the component gratings.

However, our findings in Experiment 2 demonstrated that this was not the case: neither the amplitude nor frequency of the gamma response to plaid stimuli could be characterised by the simple summation of the response to the two component gratings. For gamma amplitude we found that the response to the plaid was reduced relative to the linear sum of the two gratings, consistent with results from both primate multi-unit recordings (Busse et al., 2009), optical imaging (MacEvoy et al., 2009) and human fMRI (McDonald et al., 2012). For gamma frequency we found the reverse result: the gamma response had a higher frequency to plaids than would be expected from their response to gratings even when matched for Michelson contrast.

In previous work, Busse et al. (2009) found that population firing rates in V1 to plaid stimuli could be explained using a normalisation model in which responses of individual cells received divisive normalisation in proportion to an exponent of the root-mean-square (RMS) contrast of the stimulus. When gamma amplitude (after first being normalised across participants) in our data is plotted with respect to the RMS contrast, it can be seen that there was also a strong indication of a 1:1 relationship between the two variables (Figure 9). This would suggest that gamma amplitude to plaids might be determined by a non-linear, rather than linear, summation of the response to gratings.

However, while this re-analysis might be suggestive, we should be circumspect about drawing strong conclusions 

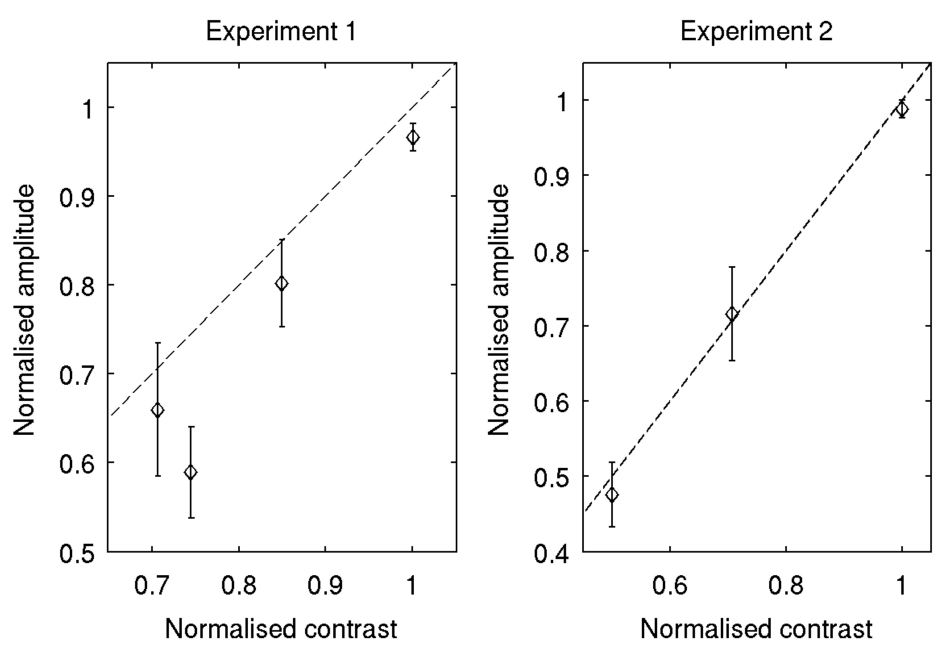

Figure 9: Plots of group mean (+/- SE) normalised gamma amplitude against normalised RMS contrast of each stimulus condition for both experiments. Gamma amplitudes in each experiment were normalised against the maximal response across conditions for each participant. RMS contrast of each condition was normalised against the maximal contrast in any condition within each experiment. In Experiment 2 the data for the grating conditions were averaged across orientations for each participant prior to normalisation. Dashed lines show $y=x$.

here. It is notable that - where sinusoidal stimuli are used at least - plaids can be perceived to be oriented differently to the component gratings (Georgeson \& Meese, 1997) and this suggests that it may not be possible to characterise responses to plaids purely in terms of the summation of their components (although we note that this effect can be reduced by making the stimuli closer to square-wave - and therefore more like the stimuli used here - by adding a $3^{\text {rd }}$ harmonic component at the appropriate phase). Instead, responses to plaids may be better characterised with respect to the presence zero crossings in the image (Georgeson \& Meese, 1997). In Experiment 2 the plaid stimulus had twice the number edges (but half the contrast at each edge) compared to the contrast-matched gratings. This difference in edge structure may more relevant to explaining the differences found in the gamma responses than the summation of stimulus energy across components. Further work is therefore required to elucidate which stimulus properties determine the gamma response to luminance-defined gratings and plaids.

\section{Effects of masking on the pattern-onset ERF}

We additionally measured the pattern-onset evoked response to our stimuli. Interestingly, in Experiment 2 this evoked component did show the pattern of responses found for firing rates by Bartolo et al. (2011), with the response to the plaid stimulus being greater than that found for the grating stimuli matched for contrast. Thus, our evidence would suggest that the amplitude patternonset ERF may more closely match firing rates than the amplitude of the gamma 'spike'.

This lack of suppression of the evoked response in the presence of a cross-orientation masks appears to contradict previous findings of suppression in human VEPs (Burr \& Morrone, 1987; Busse et al., 2009). However, we note this previous work was with temporally-modulated stimuli and measured steady-state VEPs using a frequency tagging approach to distinguish responses to the base and mask gratings. In these studies it was not possible to attribute suppression to any specific temporal component of the evoked response, and therefore those findings cannot be directly compared to the responses measured here which were specific to the initial pattern-onset component evoked by static stimuli. 


\section{Implications for cross-orientation suppression}

As outlined in the introduction, the differential effects of cross-orientation masking on firing rates and gamma amplitude found by Bartolo et al. (2011) likely reflects the effects of cross-orientation suppression, a well-known phenomenon in which the response of a cell to a grating of its preferred orientation is suppressed by the addition of a mask stimulus at a non-preferred orientation (Morrone et al., 1982). Although cross-orientation suppression was initially attributed to intra-cortical inhibition (Morrone et al., 1982, 1987), more recently it has been suggested that the effect has its origins in nonlinearities in feedforward input to the cortex, either due to synaptic depression at thalamocortical synpases (Freeman et al., 2002) or to saturating and rectifying non-linearities in the responses of geniculate relay cells that provide feedfoward input to V1 from the thalamus (Priebe \& Ferster, 2006).

Based on current-source density studies in the primate (Schroeder et al., 1991) the pattern-onset ERF most likely reflects the initial wave of feedforward EPSPs in either the granular or supra-granular layers of V1 (dependent on which surface VEP in the primate corresponds to the $\mathrm{C} 1$ component measured here), and therefore directly reflects thalamocortical inputs to V1 or is downstream of them. If cross-orientation suppression is already present in the inputs to V1 then we would expect to see its effects already apparent in the pattern-onset ERF. In fact, while in Experiment 2 we did see evidence of response saturation to increases in contrast within an orientation, the average amplitude to the plaid stimulus was only slightly (and not significantly) less than sum of the average amplitudes to the two component gratings, suggesting that any cross-orientation suppression present at this stage was weak at best.

How can this finding be reconciled with previous evidence that cross-orientation suppression is insensitive to both temporal frequency and adaptation of the mask (Freeman et al., 2002), implying a pre-cortical origin for the effect? The upper panel of Figure 6 suggests that differences in gamma amplitude in Experiment 2 occurs soon after stimulus onset (although we accept that the bandpass filtering applied to the data introduces temporal smoothing and therefore the onset of gamma suppression cannot be determined exactly). Thus, our data is consistent with finding from single cell recordings in nonhuman primates that suggest that suppression lags the onset of V1 simple cells, albeit by a fairly short interval of perhaps around $10 \mathrm{~ms}$ (Smith et al., 2006; Kimura \& Ohzawa, 2009). This might suggest that, for the static gratings used here at least, suppression is not mediated by the initial thalamo-cortical drive reflected in the patternonset ERF but by rapid cortico-cortical interactions which are reflected in the gamma response.

As noted above, firing rates in V1 to plaid stimuli have been successfully modelled by divisive normalisation based on an exponent of the RMS contrast of the stimulus (Busse et al., 2009), and in our current data the gamma amplitude appears to be linearly related to the RMS contrast of the stimulus (Figure 9). This might suggest then that gamma amplitude could reflect the activity of a population of cells generating divisive normalisation in V1. This would be consistent with previous evidence that oscillations in the gamma-band are dependent on parvalbumin-expressing GABAergic interneurons (Cardin et al., 2009) - a population of cells known to be capable of producing divisive inhibition of pyramidal cells (Atallah et al., 2012).

Interestingly, the one condition that does not appear to be well fit by RMS contrast is the $17 \%$ mask condition in Experiment 1, where gamma amplitude appears to be less than expected (Figure 9 left panel). However, the reduction of gamma in that condition would be consistent with the previously described phenomenon of crossorientation facilitation for low contrast masks (Meese \& Holmes, 2007), reinforcing the possibility that gamma amplitude may reflect the level of contrast normalisation generated by a stimulus.

Thus, we hypothesise that gamma amplitude may reflect the strength of divisive normalisation mediated by fast cortico-cortical intteractions, and that this normalisation process plays a role in cross-orientation masking effects. While this proposed mechanism clearly does not explain previous findings which provide evidence against an 
intra-cortical origin for cross-orientation suppression, we note that comparisons between monoptic and dichoptic suppression point to the existence of multiple mechanisms of cross-orientation suppression (Li et al., 2005; Baker et al., 2007) and that recent modelling of V1 has incorporated both feedforward and intra-cortical mechanisms in order to explain cross-orientation masking effects (Spratling, 2011). Furthermore, this hypothesis could be directly tested in future studies be explicitly manipulating the RMS contrast of stimuli and the distribution of stimulus energy across orientations, and by linking consequent modulations of the gamma response to psychophysical measures of cross-orientation suppression.

\section{Conclusions}

We have compared the visual gamma response to luminance-defined gratings and plaids in order to determine the possibility of distinguishing subcomponents of the gamma response in humans. We found that orthogonal masks reduced gamma amplitude but increased gamma frequency relative to a base grating (when Michelson contrast was matched across stimuli). Although to our knowledge this is the first time that this effect has been tested in humans, our data is consistent with evidence in non-human primates on the narrowband gamma response to plaids (Lima et al., 2010; Bartolo et al., 2011). However, we failed to find any evidence for a broadband subcomponent of the human gamma response that had previously reported in primates (Jia et al., 2011; Ray \& Maunsell, 2011; Hermes et al., 2014). Whether this is due to a failure of our experimental design to dissociate these components, or due to the broadband response being unable to produce a measurable magnetic field outside the head is unclear.

Our results do however have interesting implications for theories about the origins of cross-orientation suppression in primary visual cortex. Notably, against the prevailing notion that the phenomenon has its origins in geniculate inputs to V1, we failed to find evidence of crossorientation suppression in the initial pattern onset evoked response. Instead, based on the effects of cross-orientation interactions on the gamma response we have proposed a role for rapid intra-cortical processes in generating crossorientation suppression.

\section{Acknowledgements}

The author would like to thank Bethany Routley for assistance with data collection, and Krish Singh and Loes Koelewijn for useful comments on a draft version of this manuscript. This research was supported by the Cardiff University Brain Imaging Centre and the School of Psychology, Cardiff University.

\section{Abbreviations \\ EPSP - Excitatory post-synaptic potential \\ ERF - Event-related field \\ LFP - Local field potential \\ MEG - Magnetoencephalography \\ RMS - Root mean square \\ SAM - Synthetic aperture magnetometry \\ VEP - Visual evoked potential}

\section{References}

Adjamian, P., Holliday, I.E., Barnes, G.R., Hillebrand, A., Hadjipapas, A., \& Singh, K.D. (2004) Induced visual illusions and gamma oscillations in human primary visual cortex. Eur J Neurosci, 20, 587-592.

Atallah, B. V, Bruns, W., Carandini, M., \& Scanziani, M. (2012) Parvalbumin-expressing interneurons linearly transform cortical responses to visual stimuli. Neuron, 73, 159-170.

Baker, D.H., Meese, T.S., \& Summers, R.J. (2007) Psychophysical evidence for two routes to suppression before binocular summation of signals in human vision. Neuroscience, 146, 435-448.

Bartolo, M.J., Gieselmann, M.A., Vuksanovic, V., Hunter, D., Sun, L., Chen, X., Delicato, L.S., \& Thiele, A. (2011) Stimulus-induced dissociation of neuronal firing rates and local field potential gamma power and its relationship to the resonance blood oxygen level-dependent signal in macaque primary visual cortex. Eur J Neurosci, 34, 1857-1870. 
Brainard, D. (1997) The Psychophysics Toolbox. Spat Vis, 10, 433-436.

Burr, D.C. \& Morrone, M.C. (1987) Inhibitory interactions in the human vision system revealed in pattern-evoked potentials. J Physiol, 389, 1-21.

Busse, L., Wade, A.R., \& Carandini, M. (2009) Representation of concurrent stimuli by population activity in visual cortex. Neuron, 64, 931-942.

Cardin, J.A., Carlén, M., Meletis, K., Knoblich, U., Zhang, F., Deisseroth, K., Tsai, L.-H., \& Moore, C.I. (2009) Driving fast-spiking cells induces gamma rhythm and controls sensory responses. Nature, 459, 663-667.

Freeman, T.C.B., Durand, S., Kiper, D.C., \& Carandini, M. (2002) Suppression without inhibition in visual cortex. Neuron, 35, 759-771.

Georgeson, M.A. \& Meese, T.S. (1997) Perception of stationary plaids: the role of spatial filters in edge analysis. Vision Res, 37, 3255-3271.

Gieselmann, M.A. \& Thiele, A. (2008) Comparison of spatial integration and surround suppression characteristics in spiking activity and the local field potential in macaque V1. Eur J Neurosci, 28, 447459.

Hall, S.D., Holliday, I.E., Hillebrand, A., Singh, K.D., Furlong, P.L., Hadjipapas, A., \& Barnes, G.R. (2005) The missing link: analogous human and primate cortical gamma oscillations. NeuroImage, 26, 13-17.

Hermes, D., Miller, K.J., Wandell, B.A., \& Winawer, J. (2014) Stimulus dependence of gamma oscillations in human visual cortex. Cereb Cortex, advance online publication.

Huang, M.X., Mosher, J.C., \& Leahy, R.M. (1999) A sensor-weighted overlapping-sphere head model and exhaustive head model comparison for MEG. Phys Med Biol, 44, 423-440.
Jia, X., Smith, M.A., \& Kohn, A. (2011) Stimulus selectivity and spatial coherence of gamma components of the local field potential. J Neurosci, 31, 9390-9403.

Kimura, R. \& Ohzawa, I. (2009) Time course of crossorientation suppression in the early visual cortex. $J$ Neurophysiol, 101, 1463-1479.

Kleiner, M., Brainard, D., \& Pelli, D. (2007) What's new in Psychtoolbox-3. Perception 36 ECVP Abstract Supplement,.

Koelewijn, L., Dumont, J.R., Muthukumaraswamy, S.D., Rich, A.N., \& Singh, K.D. (2011) Induced and evoked neural correlates of orientation selectivity in human visual cortex. NeuroImage, 54, 2983-2993.

Li, B., Peterson, M.R., Thompson, J.K., Duong, T., \& Freeman, R.D. (2005) Cross-orientation suppression: monoptic and dichoptic mechanisms are different. $J$ Neurophysiol, 94, 1645-1650.

Lima, B., Singer, W., Chen, N.-H., \& Neuenschwander, S. (2010) Synchronization dynamics in response to plaid stimuli in monkey V1. Cereb Cortex, 20, 1556-1573.

MacEvoy, S.P., Tucker, T.R., \& Fitzpatrick, D. (2009) A precise form of divisive suppression supports population coding in the primary visual cortex. Nat Neurosci, 12, 637-645.

McDonald, J.S., Mannion, D.J., \& Clifford, C.W.G. (2012) Gain control in the response of human visual cortex to plaids. $J$ Neurophysiol, 107, 2570-2580.

Meese, T.S. \& Holmes, D.J. (2007) Spatial and temporal dependencies of cross-orientation suppression in human vision. Proc Biol Sci, 274, 127-136.

Miller, K.J., Sorensen, L.B., Ojemann, J.G., \& Den Nijs, M. (2009) Power-law scaling in the brain surface electric potential. PLoS Comput Biol, 5, e1000609.

Morrone, M.C., Burr, D.C., \& Maffei, L. (1982) Functional implications of cross-orientation 
Inhibition of cortical visual cells. I.

Neurophysiological evidence. Proc $R$ Soc Lond $B$ Biol Sci, 216, 335-354.

Morrone, M.C., Burr, D.C., \& Speed, H.D. (1987) Crossorientation inhibition in cat is GABA mediated. Exp Brain Res, 67, 635-644.

Muthukumaraswamy, S.D., Singh, K.D., Swettenham, J.B., \& Jones, D.K. (2010) Visual gamma oscillations and evoked responses: variability, repeatability and structural MRI correlates. NeuroImage, 49, 3349-3357.

Odom, J.V., Bach, M., Barber, C., Brigell, M., Marmor, M.F., Tormene, A.P., Holder, G.E., \& Vaegan (2004) Visual evoked potentials standard (2004). Doc Ophthalmol, 108, 115-123.

Pelli, D. (1997) The VideoToolbox software for visual psychophysics: Transforming numbers into movies. Spat Vis, 10, 437-442.

Perry, G., Hamandi, K., Brindley, L.M., Muthukumaraswamy, S.D., \& Singh, K.D. (2013) The properties of induced gamma oscillations in human visual cortex show individual variability in their dependence on stimulus size. NeuroImage, 68, 83-92.

Priebe, N.J. \& Ferster, D. (2006) Mechanisms underlying cross-orientation suppression in cat visual cortex. Nat Neurosci, 9, 552-561.

Privman, E., Malach, R., \& Yeshurun, Y. (2013) Modeling the electrical field created by mass neural activity. Neural Netw, 40, 44-51.

Ray, S. \& Maunsell, J.H.R. (2011) Different origins of gamma rhythm and high-gamma activity in macaque visual cortex. PLoS Biology, 9, e1000610.

Robinson, S.E. (2004) Localization of event-related activity by SAM(erf). Neurol Clin Neurophysiol, 109.

Robinson, S.E. \& Vrba, J. (1999) Functional neuroimaging by synthetic aperture magnetometry (SAM). In Yoshimoto, T., Kotani, M., Kuriki, S., Karibe, H., \& Nakasato, N. (eds), Recent Advances in Biomagnetism. Sendai, Japan: Tohoku Univ. Press, pp. 302-305.

Schroeder, C.E., Tenke, C.E., Givre, S.J., Arezzo, J.C., \& Vaughan Jr, H.G. (1991) Striate cortical contribution to the surface-recorded pattern-reversal VEP in the alert monkey. Vision Res, 31, 11431157.

Smith, M.A., Bair, W., \& Movshon, J.A. (2006) Dynamics of suppression in macaque primary visual cortex. J Neurosci, 26, 4826-4834.

Smith, S.M. (2002) Fast robust automated brain extraction. Hum Brain Mapp, 17, 143-155.

Spratling, M.W. (2011) A single functional model accounts for the distinct properties of suppres- sion in cortical area V1. Vision Res, 51, 563-576.

Swettenham, J.B., Muthukumaraswamy, S.D., \& Singh, K.D. (2009) Spectral properties of induced and evoked gamma oscillations in human early visual cortex to moving and stationary stimuli. $J$ Neurophysiol, 102, 1241-1253.

Van Pelt, S. \& Fries, P. (2013) Visual stimulus eccentricity affects human gamma peak frequency. NeuroImage, 78, 439-447.

Vrba, J. \& Robinson, S.E. (2001) Signal processing in magnetoencephalography. Methods, 25, 249-271.

Zanos, T.P., Mineault, P.J., \& Pack, C.C. (2011) Removal of spurious correlations between spikes and local field potentials. J Neurophysiol, 105, 474-486. 